\title{
LA CASA DEL PARAÍSO
}

\section{(THE HOUSE OF THE PARADISE)}

José Miguel de Prada Poole, Dr. Arquitecto

(1) Roberto Goycolea, Eduardo Navadijos y María Delgado, Arquitectos Colaboradores

Fecha de recepción: 13.1.92

RESUMEN

La memoria que se utilizó para el Concurso, al que se presentó esta propuesta, es la que sirve para su exposición.

Es ésta, una casa en la que el Hombre es amigo de la naturaleza, la controla, y la incorpora a su entorno más inmediato. Hombres, animales y plantas conviven formando un sistema interdependiente.

La vivienda, da cobijo al hombre como grupo (cualquier clase de grupo con ciertas reglas de convivencia reconocidas).

Se protege la intimidad individual mediante "elementos compactos de edificación", en los que el usuario puede aislarse por completo.

El vidrio del cerramiento es electroóptico y sus propiedades varian a voluntad.

Todas las instalaciones, controles y redes necesarios se encuentran bajo el pavimento.

(1) Primer premio en el Concurso "La vivienda del 2001", convocado por el Colegio Oficial de Arquitectos de Madrid, 1991.

\section{SUMMARY}

The article is the report that was submitted for the Contest.

This is a house in which "the man" is a friend of the nature, he controls it and incorporates it in his most immediate surroundings. People, animals and plants live together forming an interdependent system.

The house gives lodging to the man as a group (any kind of group with certain recognized rules of cohabitation).

The individual intimacy is protected through "compact building elements" in which the user can retreat and get completely isolated.

The glass of the partition is electro-optical and its properties vary as desired.

All the necessary installations, controls and networks are situated under the pavement. 


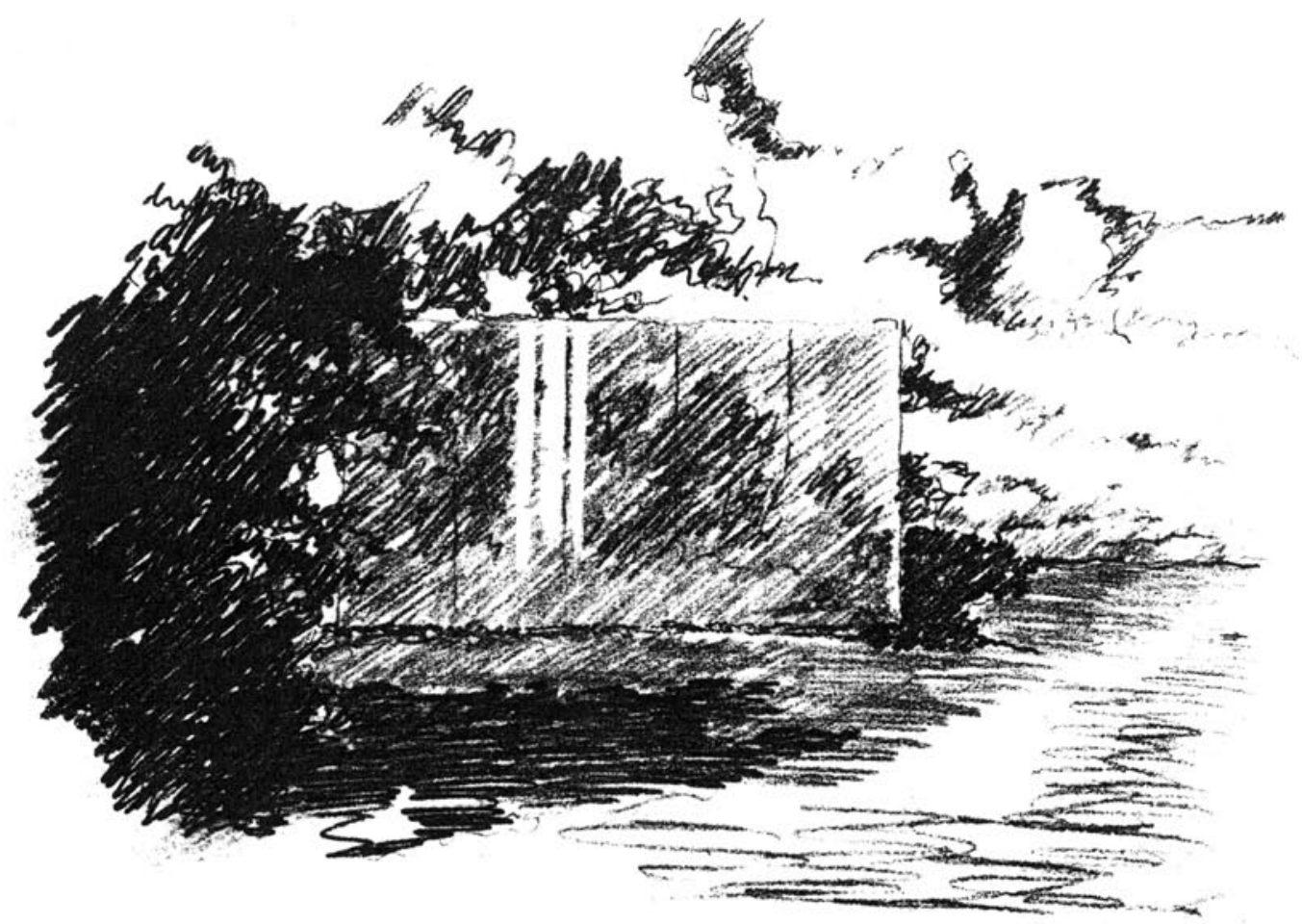

“...La naturaleza es incómoda. El césped es duro, lleno de bultos y húmedo, plagado de molestos insectos negros. Hasta el más pobre trabajador de Morris puede fabricar un asiento más confortable de lo que puede toda la Naturaleza junta. No me quejo. Si la naturaleza hubiera sido confortable el hombre nunca hubiera inventado la Arquitectura, y yo prefiero las casas que el aire libre".

Oscar Wilde.

\section{ARQUITECTURA Y NATURALEZA}

\section{Paradoja}

Arquitectura y Naturaleza se han diferenciado en su origen. La manipulación sobre el medio que supone la Arquitectura separa a ésta de la Naturaleza. (En tanto que el concepto naturaleza señale como tal todo en lo que no tenga lugar la intervención humana podríamos entender ambas palabras como contrapuestas).

Esta contraposición, entre Arquitectura y Naturaleza, ha llevado a pensar que, mundo vegetal y animal, que existían antes que el hombre, $y$ han formado parte desde siempre de la Naturaleza, son su oponente; mundos antagónicos del humano con los que hay que mantener las distancias.

Y esta contraposición ha dado también origen a una paradoja: Ya que el hombre es un ser técnico, cuya supervivencia se cifra en manipular el entorno para poder sobrevivir, "dada su naturaleza técnica" tiene que ser enemigo de la Naturaleza. Conclusión que parecería avalada por la observación de las consecuencias que sobre el entorno ha tenido la aplicación indiscriminada de dicha técnica. Pero dado que el hombre procede de la Naturaleza y forma parte de ella, dependiendo de ella para su subsistencia, debe así mismo ser su amigo y protegerla para asegurar tanto su supervivencia como el fundamento de su propio ser, su ser natural. 


\section{Propuesta}

Conclusión: La ciudad, y por lo tanto la habitación, como producto artificial de la "tekné" se apartan de la Naturaleza, pero el hombre por su esencia tiende inevitablemente a ella. ¿Cómo vivir socialmente en la naturaleza?

Si se admite que Naturaleza es todo aquello en lo que no ha intervenido la mano del hombre, esta conclusión será un desgarrador dilema sin solución. Pero si se admito Niı natırala>a ac tamhión todn ariol nrodiıntn
El primer objetivo teórico de nuestro proyecto ha sido el mostrar que el binomio técnica-naturaleza lejos de expresar dos términos opuestos, nos debería indicar que la única manera de resolver los problemas existentes de nuestra relación con la naturaleza pasa por el empleo a fondo de nuestro conocimiento para aplicar las técnicas adecuadas de protección y desarrollo de nuestro entorno tanto animal como vegetal. 

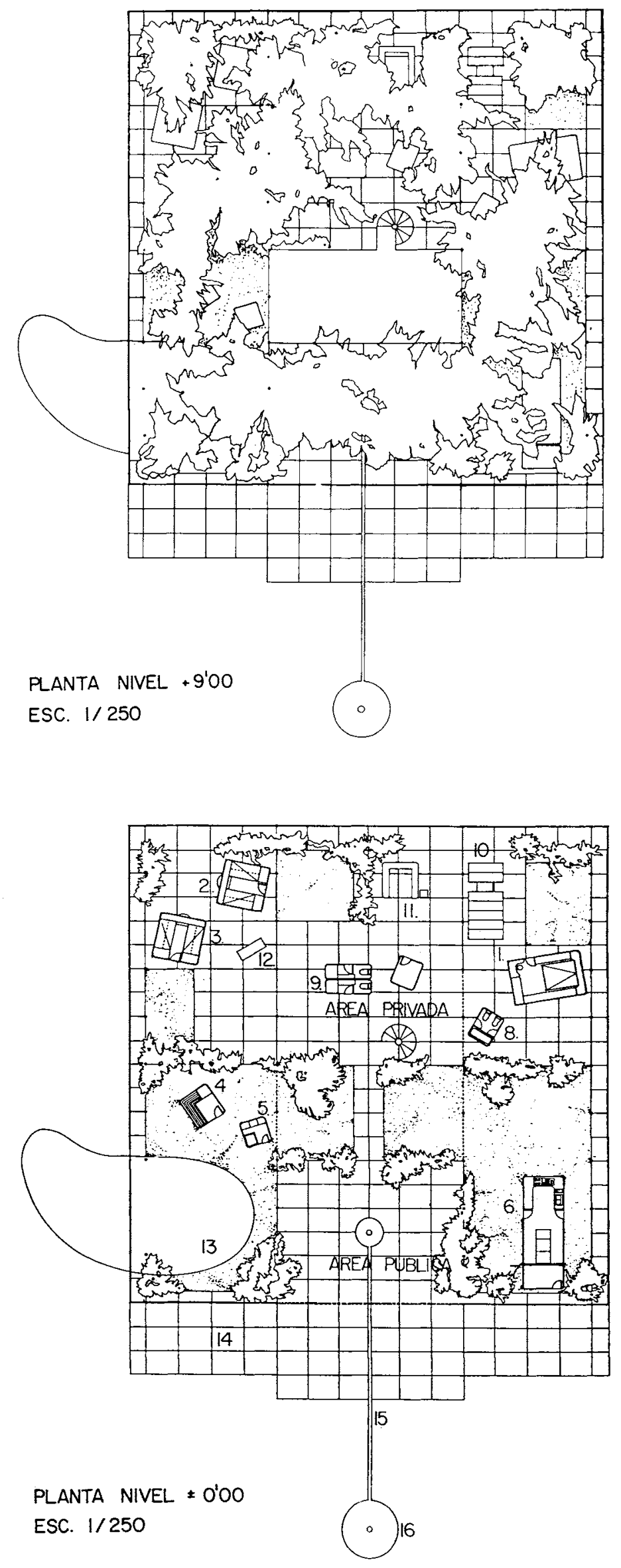


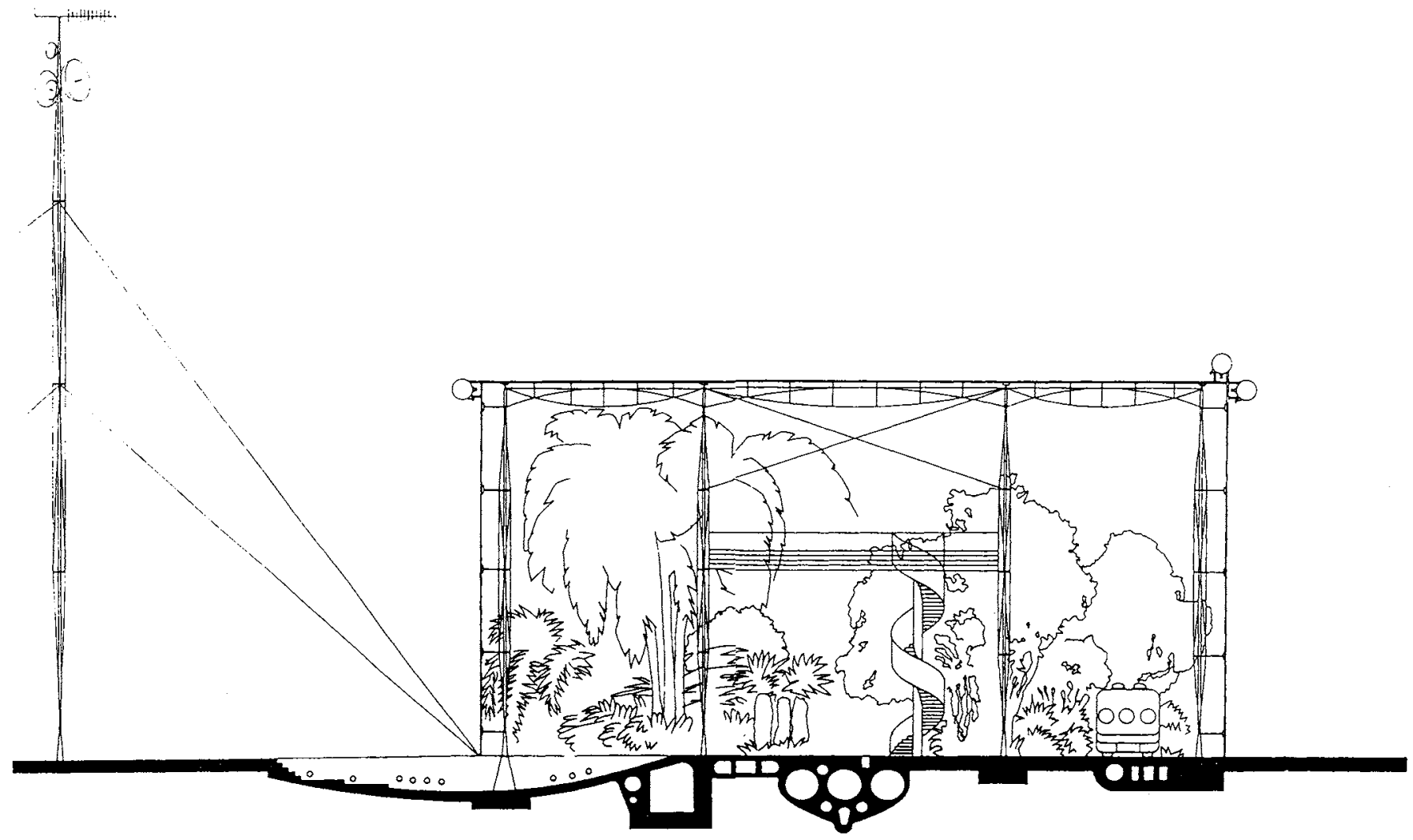

\section{LEYENDA}
1. MÓDULO REPOSO
2. MÓDULO REPOSO
3. MÓDULO REPOSO
4. SAUNA
5. VESTUARIO
6. MÓDULO ALIMENTACIÓN
7. MÓDULO LIMPIEZA
8. MÓDULO ASEO
9. MÓDULO ASEO
10. INFOTECA - VIDEOTECA
11. INFORMATICA
12. MÓDULO BRICOLAGE
13. ESTANQUE / PISCINA
14. SOLARIUM
15. ARROYO
16. FUENTE

\section{INDIVIDUO Y SOCIEDAD}

\section{Paradoja}

Otra de las aparentes contradicciones que fustigan a nuestra sociedad es el énfasis en la conciencia de individualización. El individuo se siente "diferente" al otro, y esta diferencia crea antagonismos. Se siente uno por oposición a muchos; por oposición a una sociedad cuyo perfil se hace vago y se difumina. El individuo frente a la sociedad lejos de aceptarla como parte de sí mismo, al igual que ocurria en la "polis" griega, se limita a reclamar de ella sus derechos sin admitir sus servidumbres.

Frente al grupo familiar sucede algo análogo. La tendencia es que la familia se desintegra, primero en familia nuclear, y posteriormente en individuos aislados, que viven la vida personal independientemente de cualquier tipo de grupo. Por ello, en las sociedades más desarrolladas aumenta dramáticamente el número de viviendas ocupadas por una sola persona. 


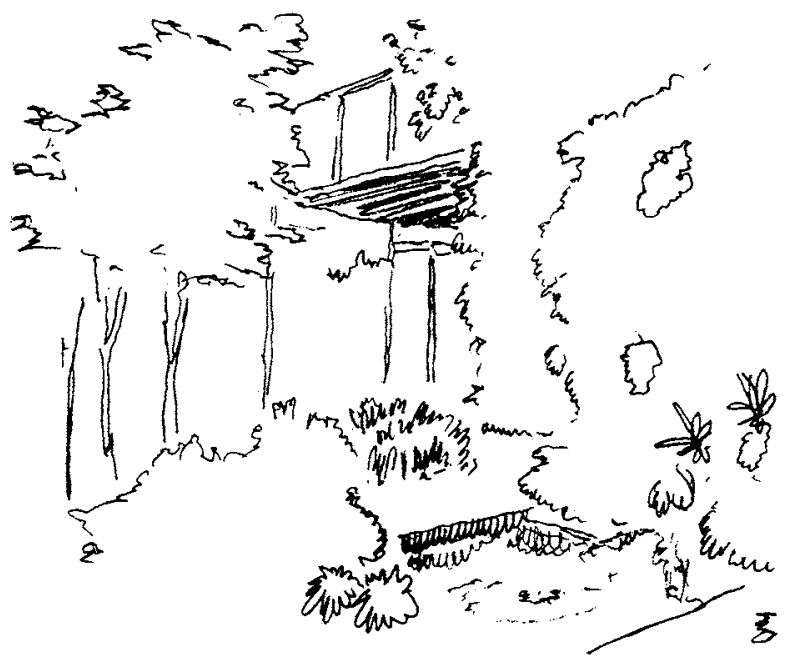

Pero como contrapartida el hombre es básicamente un ser social. El propio desarrollo individual no tiene sentido $\sin$ la sociedad que define su forma de ser individual. El individuo autónomo no existe más que como curiosidad. Todos los logros de conocimiento, cultura, supervivencia y bienestar, proceden de la existencia de un cuerpo social. Pero si individuo y grupo, persona y sociedad, fuesen conceptos antagónicos, ¿cómo superar la esquizofrenia de semejante situación?

No hay tal dilema. Es otra falacia más que procede del malentendimiento de un concepto de sociedad, derivado de una idea degradada por sociedades particulares cuyo funcionamiento es anómalo o insatisfactorio, y por lo tanto no aceptado.

\section{Propuesta}

En este proyecto suponemos que de momento no existen sociedades ideales, pero suponemos también que una acertada consciencia individual, imbuida del ser social, sí puede producir individuos sociales cuya característica principal sea la aceptación, tanto de los derechos como de las servidumbres de sociedades insatisfactorias que va ser posible transformar, no tanto por la acción individual como por la acción conjunta del grupo social.

Por ello, nuestra vivienda dará cobijo en primer lugar al grupo, sea éste familiar, de convivencia, de intereses, de ideas, de afinidades o pasiones tipo falansterio, etc. (para nosotros esto es irrelevante mientras se trate de un grupo social coherente), en el que la aceptación de las reglas de convivencia sea asumida con todas sus consecuencias.

Protegemos la intimidad individual mediante "compactos", en los que el usuario puede aislarse por completo del grupo general, o pertenecer a un grupo más restringido de dos o tres personas (pareja más niño, por ejemplo).

\section{Tecnología}

La vivienda se organiza como un espacio-jardín que podríamos denominar como de clima protegido. La sensación es la de vivir al aire libre en una zona de clima privilegiado, de zona templada o subtropical. Se plantea como un invernadero-veraneadero avanzado, preparado para proteger el interior de todo tipo de situaciones climáticas que se alejen de los niveles máxi- 
mos y mínimos de confort establecidos previamente. Tendriamos así tres ambientes climáticos distintos del clima exterior:

a) Un clima general atemperado creado por la membrana de protección, la vegetación y las láminas de agua.

b) Un ambiente de clima a nivel de suelo 1,8 $\mathrm{m}$ de altura, de clima reforzado, en el que se utilizan las zo-

\section{Cerramiento / Protección}

El vidrio de protección es un vidrio electro-óptico cuyas propiedades cambian al variar un campo eléctrico asociado, pudiendo oscurecerse casi por completo (existente como producto experimental). La estructura soporte de esta envolvente es muy ligera, empleando recursos ahora conocidos puntualmente que pronto estarán muy difundidos y serán sumamente económicos, a base de cables anticlásticos que permiten el trabajo a tracción tanto cuando la carga es positiva como cuando es negativa.

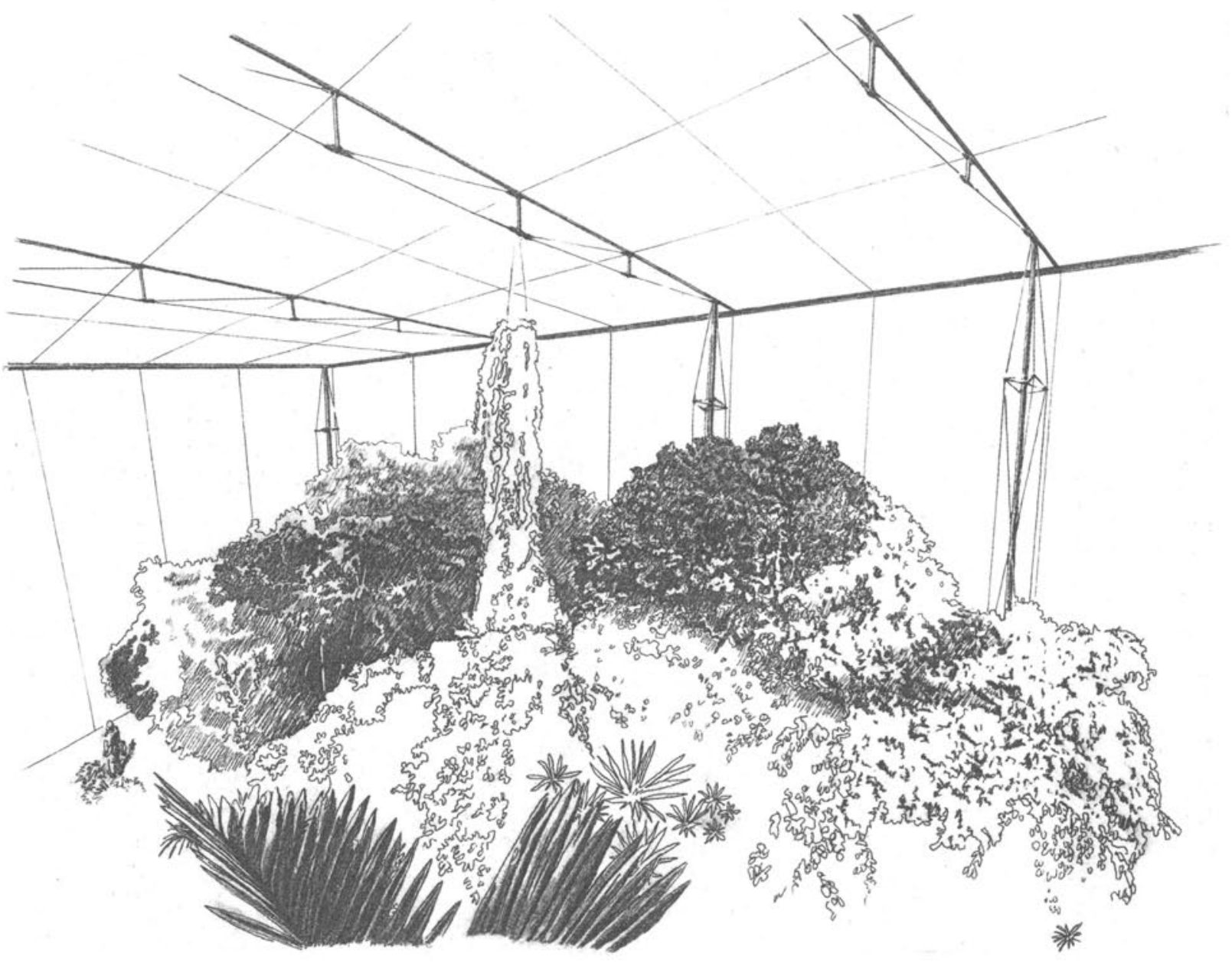

Centro de Control e Instalaciones

nas pavimentadas como acumuladores térmicos tanto en invierno (de calor: $29 \div 32^{\circ}$ ) como en verano (de frío: $20 \div 24^{\circ}$ ), así como se fuerza a circular el aire en un sentido determinado con bocas de im. pulsión convenientemente distribuidas a nivel del pavimento, creando una brisa de $2 \div 3 \mathrm{~m} / \mathrm{s}$ para incrementar la sensación de confort y facilitar la evapotranspiración de las plantas.

c) Un clima individual especifico en los "compactos", logrado por medios tradicionales de refrigeración/ calefacción.
El centro de control, instalaciones de depuración, digestores, aprovechamiento de metano, y baterias de acumulación de energía fotovoltaica de las células de silicio de la cubierta se hallan en sótano. Bajo el pavimento se hallan también las redes de distribución de energía eléctrica, gas, agua, ventilación, aire a alta y baja presión, y circuito automático de riego y nutrientes para la vegetación. Los circuitos de informática y telefónicos son inalámbricos, así como los de televisión y radio para los que existe una conexión exterior vía satélite. 

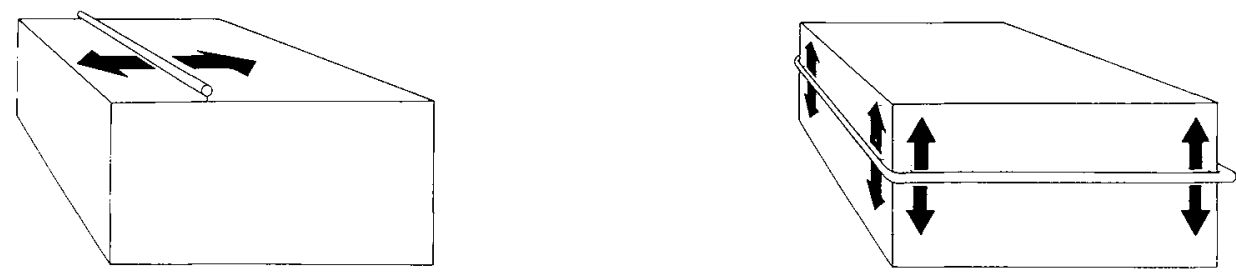

Sistema de limpieza exterior de cerramiento, a base de agua a presión con conductos móviles

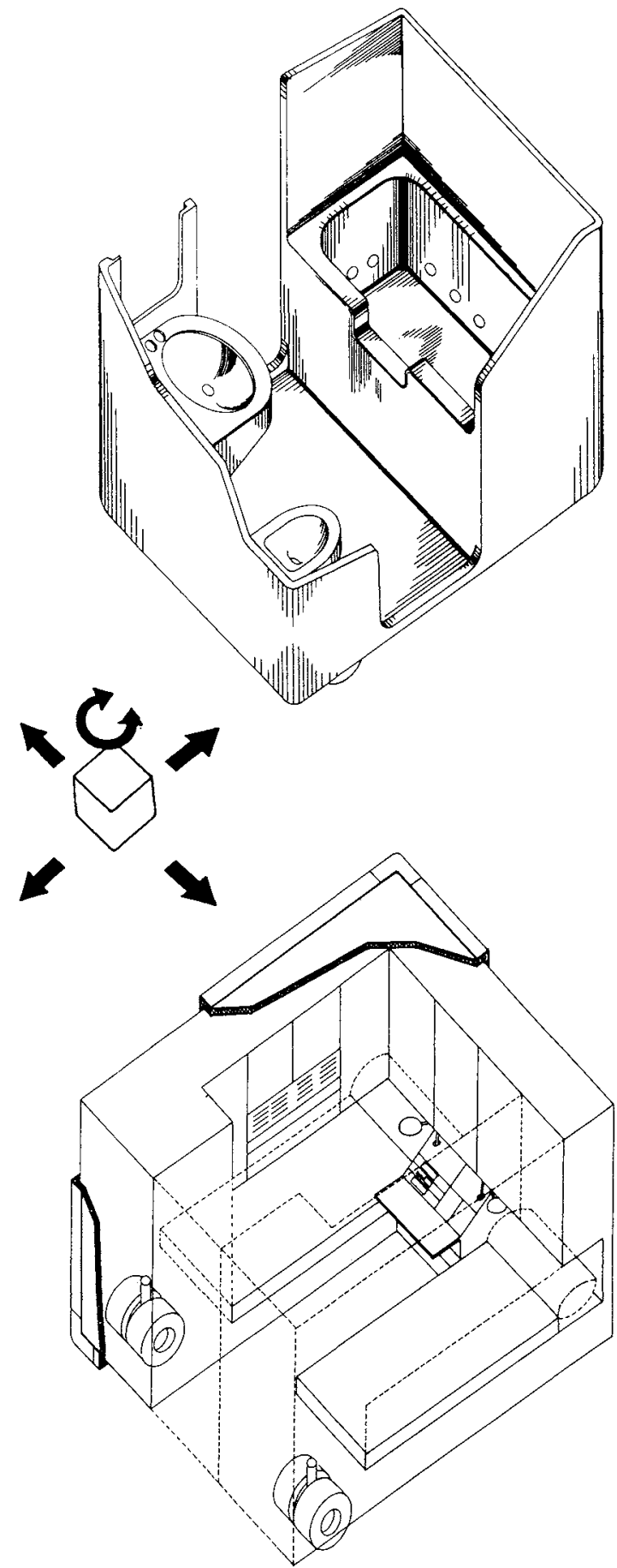

\section{Distribución}

Según se puede observar en la planta la distribución, está de tal manera que las diversas áreas de influencia o de uso se solapan una sobre otras sin estable. cer conflictos. Así podremos advertir que la mitad primera es un área de relación social intergrupos, mientras que la mitad posterior lo es de relación interindividual. Que hay áreas de reposo y de actividad, que a su vez puede ser intelectual (biblioteca, informática y telemática) o física ("hobbies" o deportes). Áreas de tráfico intenso y áreas de remanso, que coinciden con las de descanso, etc.

\section{"Compactos" de actividades específicas}

Existen varios tipos de "compactos" que guardan relación con las áreas anteriores, que se pueden dividir en dos grupos:

A) Que encierran actividad de personas, o

B) Que guardan cosas.

En el primer caso encontramos los de dormitorio, estudio o aislamiento individual, aseo, sauna y vestuario, y preparación de alimentos.

En el segundo caso, todos los de almacenamiento:

a) de ropa, coleccionismo, etc, que son independientes y no necesariamente forman parte de los "compactos de habitación".

b) de "hobbies" o mantenimiento, así hay "compactos taller" de: modelos, carpintería, jardineria, etc,

c) y de servicios en general, como el módulo de despensa o alimentos y el de lavanderia y clasificación de desperdicios.

Los "compactos" tienen la particularidad de ser elementos móviles, lo que permite situarlos en cualquier emplazamiento dentro de la vivienda, de acuerdo a los gustos y/o necesidades del momento. 


\section{Entorno}

Por último, toda el área del entorno próximo a nuestro "microclima" forma también parte sustancial del mismo, pues contribuye eficazmente a crear un "colchón protector" a modo de abrigo que prepara el aire exterior frenándolo y acondicionándolo para que las pérdidas e intercambios sean los menores posibles.

La influencia de nuestra habitación se derrama, por así decirlo, por los alrededores extendiendo su benéfica influencia y cuidado, y contribuyendo de esta manera a crear naturaleza, que a largo plazo y merced al esfuerzo particular de miles de grupos sociales terminarían transformando el paisaje desértico en un medio amable en el que la vida fuera fácil.
La vivienda carece de divisiones o tabiques. Barreras vegetales cortan las vistas y amortiguan la transmisión de ruidos, creando áreas o dominios de actividades, que permiten una interrelación continua del individuo con el grupo general con el que comparte la responsabilidad del cuidado y mantenimiento tanto de su zona de influencia como del sistema en su totalidad. A su vez, y como equilibrio entre grupo e individuo, cada una de las zonas, así como cada uno de los "compactos" que pueden cambiar de lugar con muy pocas limitaciones, pueden funcionar de manera autónoma teniendo relaciones independientes con el exterior.

Madrid, junio 1991

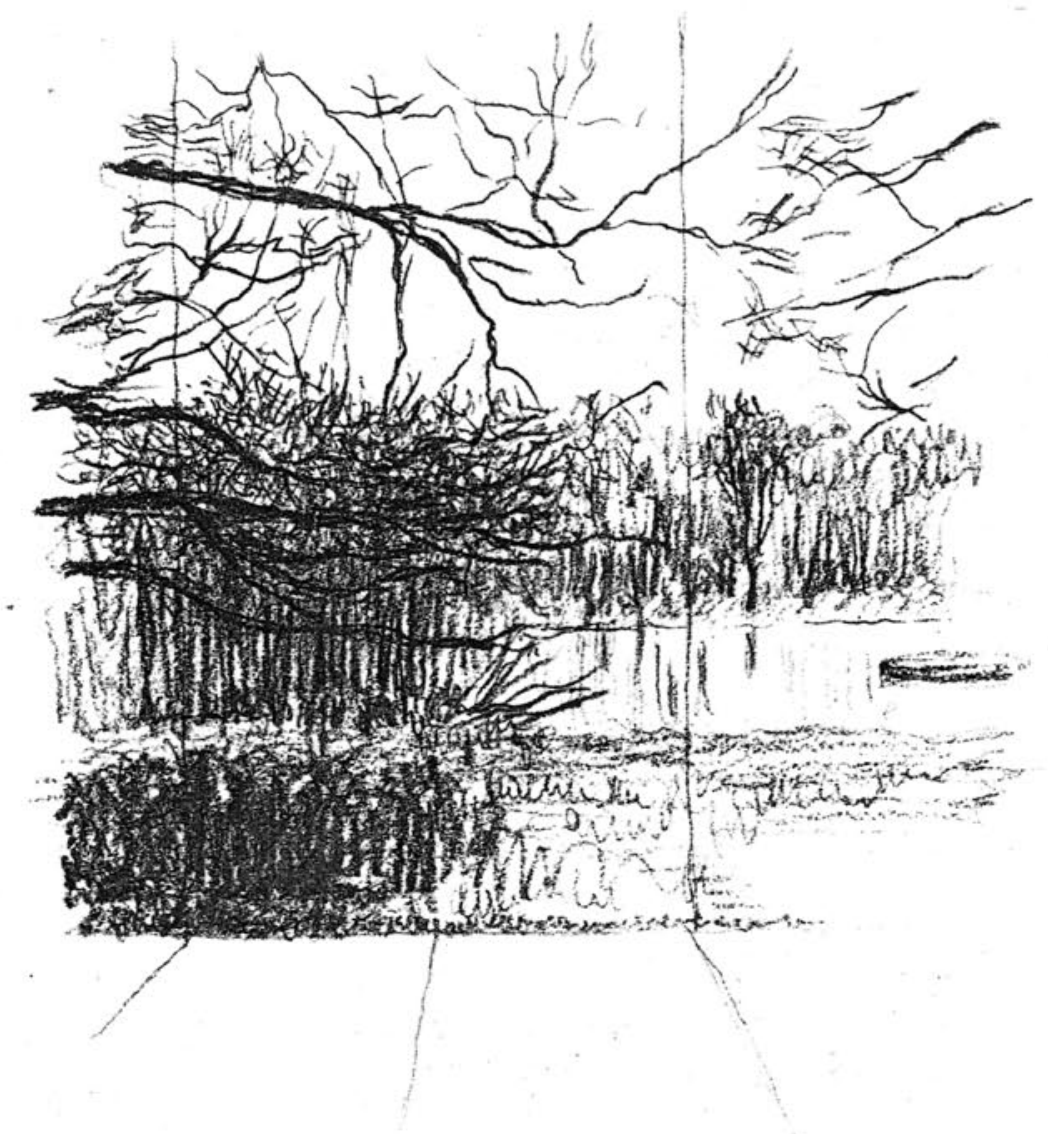

